\title{
MASSIVELY PARALLEL FOKKER-PLANCK CALCULATIONS - EPILOGUE
}

\author{
Arthur A. Mirin \\ National Energy Research Supercomputer Center \\ Lawrence Livermore National Laboratory \\ October 1990
}

The purpose of this writeup is to supplernent the publication, "Massively Parallel Fokker-Planck Calculations," which appeared in the Proceedings of the Fifth Distributed Memory Computing Conference[1]. Results obtained subsequent to that presentation are reported. This work is not self-contained; the reader should refer to Reference [1].

\section{Comparison of Connection Machines}

The timings presented in Reference [1] were those using the Connection Machine at NASA Ames. Unless otherwise stated, the timings presented here are for the Connection Machine it the Advanced Computing Laboratory $(\mathrm{ACL})$ at Los Alamos National Laboratory. A comparison for the fine mesh case is shown in Table 1.

Table 1. Comparison of Connection Machines for Fine Mesh Case

\begin{tabular}{|c|c|c|c|c|}
\hline \multirow[b]{2}{*}{ Procedure } & \multicolumn{2}{|c|}{ NASA } & \multicolumn{2}{|c|}{$\underline{\mathrm{ACL}}$} \\
\hline & CM-total & CM-active & CM-total & CM-active \\
\hline nts & $3.0 \times 10^{-2}$ & $2.8 \times 10^{-2}$ & $4.6 \times 10^{-2}$ & $2.2 \times 10^{-2}$ \\
\hline Time & $1.0 \times 10^{-2}$ & $1.0 \times 10^{-2}$ & $1.3 \times 10^{-2}$ & $1.1 \times 10^{-2}$ \\
\hline
\end{tabular}

The CM at NASA Ames has 32-bit hardware, whereas the CM at the ACL has 64-bit hardware. Note the difference in the CM-active times. Even though both computations are single precision, the 64-bit hardware executes differently from the 32-bit hardware. Because of the difficulty in performing accurate $C M$ timings, the difference in the CM-total times might be due to differences in the loads on the respective systems.

\section{Comparison of Front Ends}

The CM Fortran compiler is now available on the Sun-4 front end. A comparison of the Vax and Sun front ends is shown in Table 2. 
Table 2. Comparison of Front Ends for Fine Mesh Case

\begin{tabular}{|c|c|c|c|c|}
\hline \multirow[b]{2}{*}{ Procedure } & \multicolumn{2}{|c|}{ VAX } & \multicolumn{2}{|c|}{ SUN } \\
\hline & CM-total & CM-active & CM-total & CM-active \\
\hline & $4.6 \times 10^{-2}$ & $2.2 \times 10^{-2}$ & $2.2 \times 10^{-2}$ & $1.9 \times 10^{-2}$ \\
\hline & $1.3 \times 10^{-2}$ & $1.1 \times 10^{-2}$ & $1.1 \times 10^{-2}$ & $1.0 \times 10^{-2}$ \\
\hline
\end{tabular}

It can be seen that the Sun front end is superior. The variation in CM-total time is presumably due to differences in machine performance and load. The variation in the CM-active time is a manifestation of the inaccuracy of the timing procedure.

\section{Single versus Double Precision}

Sixty-four 'it hardware is now available on the ACL Connection

Machine. A comparison of accuracy and timing is shown in Table $j$.

The Sun- 4 front end is used.

Table 3. Standard Calculation versus Full Double Precision for Fine Mesh Case. (The initial density and energy are $6.8 \times 10^{13}$ and 49.2228 , respectively.)

Accuracy

\begin{tabular}{llc} 
Precision & Final density & Final energy \\
\cline { 2 - 2 } & $7.65638 \times 10^{13}$ & 45.9036 \\
(64-bit CM) & $7.64610 \times 10^{13}$ & 45.9133 \\
Double & $7.64600 \times 10^{13}$ & 45.9139 \\
Cray & $7.64600 \times 10^{13}$ & 45.9139
\end{tabular}

Timings

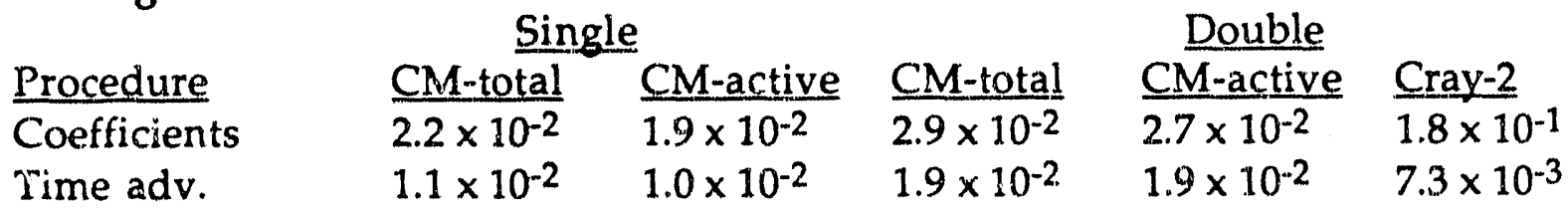


It can be seen that the double precision (64-bit) calculation takes less than twice as long as the single precision calculation. The actual arithmetic executes at the same speed, but for the 64-bit case the memory fetches and communications take twice as long. The single precision calculation executes more accurately using the 64-bit hardware because of the availability and utilization of the extra bits during the arithmetic operations.

\section{Optimized Tridiagonal Solve}

A routine to solve parallel tridiagonal systems is now available in the Connection Machine Scientific Software Library (CMSSL). Like the Fortran routine used in the above cases, the CMSSL routine uses cyclic reduction; however it is my understanding that it uses ordinary rather than parallel cyclic reduction, because of a savings in communications costs. A comparison in performance is shown in Table 4. The Sun front end is used.

Table 4. Comparison of Tridiagonal Solvers for Fine Mesh Case

Procedure

Fortran

CMSSL

\begin{tabular}{ll} 
CM-total & \multicolumn{2}{l}{ Single } \\
\hline $1.1 \times 10^{-2}$ & $\frac{\text { CM-active }}{1.0 \times 10^{-2}}$ \\
$4.9 \times 10^{-3}$ & $3.9 \times 10^{-3}$
\end{tabular}

\begin{tabular}{|c|c|c|}
\hline \multicolumn{3}{|c|}{ Double } \\
\hline M-tota & CM-active & Cra \\
\hline & 1.9 & \\
\hline
\end{tabular}

It can be seen that the CMSSL routine executes roughly $60 \%$ faster than the Fortran routine.

\section{Multiple Ionic Species}

The original CM version of FPPAC was limited to one general species due to compiler limitations having to do with the alignment of multiply dimensioned arrays. With these limitations having been removed, FPPAC may now be used to execute multispecies cases on the CM.

For a Deuterium-Tritium case using the fine mesh, the accuracy and timing versus precision and front end is similar to the single species case. Results are shown in Table 5. The Sun front end and the CMSSL tridiagonal solve are implemented. 
Table 5. Two-Species Deuterium-Tritium Problem

\begin{tabular}{|c|c|c|c|c|c|}
\hline \multirow[b]{2}{*}{ Procedure } & \multicolumn{2}{|c|}{ Single } & \multicolumn{2}{|c|}{ Double } & \multirow[b]{2}{*}{ Cray-2 } \\
\hline & CM-total & CM-active & CM-total & CM-active & \\
\hline & $4.1 \times 10^{-2}$ & $3.8 \times 10^{-2}$ & $5.9 \times 10^{-2}$ & $5.5 \times 10^{-2}$ & 10 \\
\hline & $8.3 \times$ & $10^{-3}$ & $1.5 \times 10^{-2}$ & $1.4 \times 10^{-2}$ & \\
\hline
\end{tabular}

It can be seen that using double precision on the CM, the coefficients computation executes 6 times faster on the CM than on the Cray-2, and the time-advancement executes at roughly the same speed.

\section{Toward the Future}

FPPAC is a relatively simple but representative Fokker-Planck code, whereas a state-of-the-art Fokker-Planck code such as CQL3D[2] includes averaging over the particle trajectory (bounce-averaging) and radial (flux-surface) dependence. The higher dimensionality allows an even higher degree of parallelism. Hence the time-advancement, when using an alternating direction method, should execute quite favorably on the CM. Many problems demand a greater degree of implicitness and could make good use of a parallelized preconditioned conjugate gradient (PCG) technique; such solvers for the $\mathrm{CM}$ are a topic of current research. The biggest question regarding the v:ability of nonlinear Fokker-Planck calculations on the CM is whether or not the coefficients computation could be sped up for cases involving a small to moderate number of Legendre polynomials.

Present day Fokker-Planck calculations typically involve the application of radio frequency waves. The algorithms used to compute the actual ray tracing and the resulting if diffusion coefficients are both highly parallel, so that a state-of-the-art code such as CQL3D is likely to run well when ported to a massively parallel environment.

\section{Summary}

The Connection Machine is advantageous for fine resolution problems with extensive Legendre polynomial expansions. For the fine mesh case reported here, the coefficients computation executes 6 times faster (using 64-bit arithmetic) on the Connection Machine than on the Cray-2, and the timeintegration executes at roughly the same speed. Assuming a Connection Machine to cost roughly one quarter as much as a Cray-2, this translates into 
factors of 24 and 4 cost-performance in favor of the CM for the coefficients and time-advancement, respectively. At coarser resolution the cost-performance of the time-advancement is still likely to be favorable on the CM; however for short Legendre series the present implementation of the coefficients computation executes much too slowly on the $\mathrm{CM}$ and is much better suited to the Cray.

\section{References}

[1] A.A. Mirin, "Massively Parallel Fokker-Planck Calculations," Proc. Fifth Distrib. Memory Computing Conf., Charleston (1990), IEEE Computer Society Press, I, 426.

[2] M.G. McCoy and G.D. Kerbel, Comput. Phys. Commun. 40, 115 (1986). 

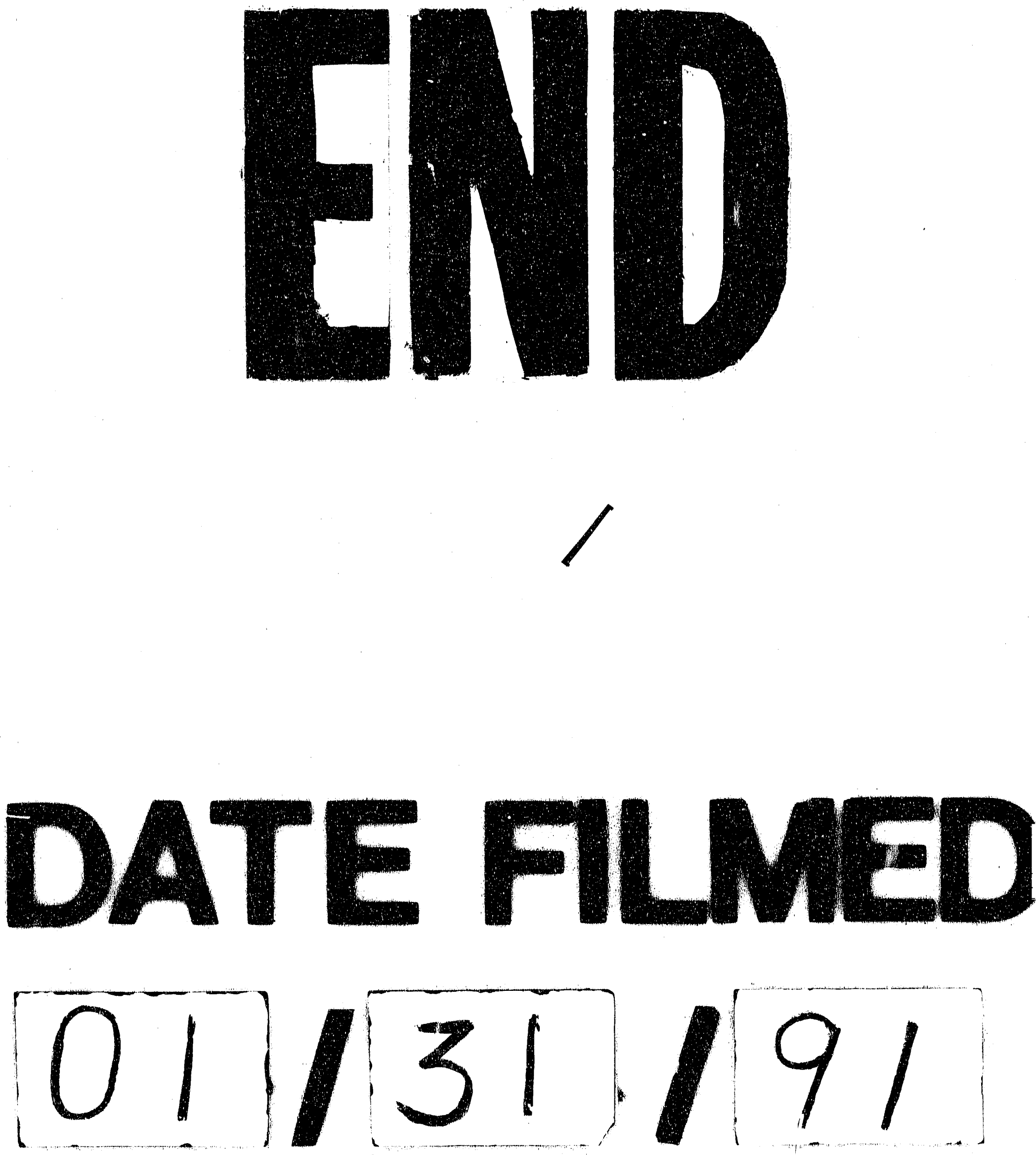
